\title{
Erratum to: Advantages of FDG-PET/CT over CT alone in the preoperative assessment of lymph node metastasis in patients with esophageal cancer
}

\author{
Ryuichi Karashima $\cdot$ Masayuki Watanabe $\cdot$ Yu Imamura $\cdot$ \\ Satoshi Ida $\cdot$ Yoshifumi Baba $\cdot$ Shiro Iwagami $\cdot$ Yuji Miyamoto \\ Yasuo Sakamoto $\cdot$ Naoya Yoshida $\cdot$ Hideo Baba
}

Published online: 18 February 2015

(C) Springer Japan 2015

Erratum to: Surg Today (2014)

DOI 10.1007/s00595-014-0965-6

Two inaccurate technical descriptions appeared in above titled article.

In the "Imaging studies" subsection, the dose of intravenously injected ${ }^{18} \mathrm{~F}-\mathrm{FDG}$ was misdescribed as $370 \mathrm{MBq}$ $(10 \mathrm{mCi})$. Actual dose of ${ }^{18} \mathrm{~F}-\mathrm{FDG}$ was $185-296 \mathrm{MBq}$ (5-8 mCi). In addition, injection rate of nonionic contrast medium during CT imaging was misdescribed as $2.5 \mathrm{ml} / \mathrm{s}$, and accurate injection rate was $2.0 \mathrm{ml} / \mathrm{s}$.

The authors sincerely apologize for these errors.

The online version of the original article can be found under doi:10.1007/s00595-014-0965-6.

R. Karashima · M. Watanabe · Y. Imamura · S. Ida · Y. Baba ·

S. Iwagami · Y. Miyamoto $\cdot$ Y. Sakamoto $\cdot$ N. Yoshida $\cdot$ H. Baba

Department of Gastroenterological Surgery, Graduate School

of Medical Sciences, Kumamoto University, Kumamoto, Japan

M. Watanabe ( $\bowtie)$

Department of Gastroenterological Surgery, Cancer Institute

Hospital of Japanese Foundation for Cancer Research,

3-8-31 Ariake, Koto-ku, Tokyo 135-8550, Japan

e-mail: masayuki.watanabe@jfcr.or.jp 